\title{
Ectopic Expression
}

National Cancer Institute

\section{Source}

National Cancer Institute. Ectopic Expression. NCI Thesaurus. Code C19566.

Expression in an abnormal part of the body 